\title{
Mir-144-3p Promotes Cell Proliferation, Metastasis, Sunitinib Resistance in Clear Cell Renal Cell Carcinoma by Downregulating ARID1A
}

\author{
Wen Xiao ${ }^{a}$ Ning Lou Hailong Ruan $^{\mathrm{a}}$ Lin Bao ${ }^{\mathrm{a}}$ Zhiyong Xiong ${ }^{\mathrm{a}}$ \\ Changfei Yuan $^{\mathrm{a}}$ Junwei Tong ${ }^{\mathrm{a}}$ Guanghua Xu $\mathrm{Yali}^{\mathrm{a}}$ Zhou ${ }^{\mathrm{b}}$ Yan Qu ${ }^{\mathrm{b}}$ \\ Wenjun-Hub Yaoying Gao ${ }^{b}$ Zeyuan Rub Lei Liu ${ }^{a}$ Haibing Xiao ${ }^{a} \quad K^{b} C^{a} n^{a}$ \\ Hongmei Yang ${ }^{\mathrm{b}}$ Xiaoping Zhang ${ }^{\mathrm{a}}$ \\ aDepartment of Urology, Union Hospital, Tongji Medical College, Huazhong University of Science \\ and Technology, Wuhan, bDepartment of Pathogenic Biology, School of Basic Medicine, Huazhong \\ University of Science and Technology, Wuhan, China
}

\section{Key Words}

Mir-144-3p • Clear cell renal cell carcinoma • OncomiRNA • ARID1A • Chemoresistance

\begin{abstract}
Background/Aims: We previously performed microRNA (miRNA) microarray to identify effective indicators of clear cell renal cell carcinoma ( $c C R C C)$ tissue samples and preoperative/ postoperative plasma in which we identified miR-144-3p as an oncomiRNA. However, the molecular mechanism of miR-144-3p remains unclear. This study aims to explore the roles of miR-144-3p in the invasion, migration and Sunitinib-resistance in $c c R C C$ and to elucidate the underlying mechanisms. Methods: Gain and loss of function approaches were used to investigate the cell proliferation, cycle distribution, clonogenicity, migration, invasion, chemosensitivity of miR-144-3p in vitro. The xenograft model was used to assess the effects of miR-144-3p overexpression on tumorigenesis. Bioinformatics analysis and dual-luciferase reporter assay were used to indentify AT-rich interactive domain 1A (ARID1A) as a direct target gene of miR-144-3p. Quantitative RT-PCR, Western blotting, and immunohistochemical (IHC) staining were used to explore ARID1A expression level of the mRNA and protein. Results: We found that miR-144-3p overexpression enhanced cell proliferation, clonogenicity, migration, invasion, and chemoresistance in ccRCC cells. Notably, the oncotumor activities of miR-144-3p were mediated by repressing the expression of ARID1A. The downregulation of ARIDIA could promote the function of miR-144-3p in cell proliferation, metastasis and chemoresistance. Consistently, ARID1A mRNA and protein levels were decreased in cCRCC and in nude mice, and they negatively correlated with miR-144-3p. Conclusion: Higher miR-144-3p may enhance
\end{abstract}

W. Xiao and N. Lou contributed equally to this study.

Xiaoping Zhang

and Hongmei Yang
Department of Urology, Union Hospital, Tongji Medical College, Huazhong University of Science and Technology, 1277 Jiefang Avenue, Wuhan, Hubei Province, (China)

Tel. 86-18602752025; Fax 85776343; E-Mail xzhang@hust.edu.cn/hyang@hust.edu.cn 
malignancy and resistance to Sunitinib in cCRCC by targeting ARID1A, the observations may uncover novel strategies of ccRCC treatment.

\section{Introduction}

Renal cell carcinoma (RCC), constituting $3.79 \%$ of all adult malignancies, is the most lethal urological malignancy with about 63, 990 new cases and 14, 440 deaths estimated for 2017 in the United States [1], with about 66, 800 new cases and 23, 400 deaths estimated for 2015 in China [2]. RCCs are a group of chemotherapy-resistant diseases that can be distinguished by histopathological features and underlying gene mutations [3, 4].

Clear cell renal cell carcinoma (ccRCC), the most common RCC histological subtype, which has the highest rate of mortality, invasion, metastasis and chemoresistance $[5,6]$. Several studies have shown that is closely associated with a series gene mutations such as TP53, VHL, PBRM1, ARID1A, PTEN, SDHB in cancer tissues [7-9], as well as TP53, VHL, NF1, ARID1A, PTEN mutation when treated with first-line and later line vascular endothelial growth factor (VEGF) tyrosine kinase inhibitors (sunitinib, pazopanib, axitinib, sorafenib) in circulating tumor DNA $[10,11]$. ARID1A is one of the commonest mutant genes and has equal frequencies when treated with first-line versus postfirst-line regimens in ccRCC [10], and it may play a key role in chemoresistance. At the same time, the lower protein of ARID1A represents a worse prognosis of clear cell renal cell carcinoma [12, 13], since ARID1A plays a role of tumor suppressor in various cancers such as ovarian cancer [14], lung adenocarcinoma [15], pancreatic cancer [16], and liver cancer [17]. However, the mechanism involved in ARID1A suppression in ccRCC remains unclear.

MicroRNAs (miRNA), first discovered in 1993 [18], are small, non-coding RNA molecules that have been known to contribute to multiple cellular processes and have been shown to influence diversified tumorigenic steps in cancers, such as proliferation $[19,20]$, apoptosis [21], metastasis [22, 23], radiation sensitivity [24] and chemosensitivity [25]. miRNAs may act as tumor suppressors or oncogenes (oncomiRs) by altering chromatin structure or by regulating the transcription of protein-coding genes [26, 27]. Mature miRNA negatively regulates gene expression through degrading the target mRNA to suppress gene translation or degradation by interacting with the target mRNA $[28,29]$. More recently, several articles confirmed the role of miRNAs as tumor biomarkers. It has been demonstrated clearly that miRNAs stably exist in plasma [30] and the regulation of circulating miRNAs was closely associated with cancer types and disease progression [31].

We previously identified miR-144-3p as the most differentially expressed miRNA in plasma microarray analysis. The plasma miR-144-3p levels significantly decreased after surgery in ccRCC patients [32]. Furthermore, the miR-144-3p levels in ccRCC tumor tissues obviously increased when compared with adjacent normal tissues in tissues microarray analysis and ccRCC tissues [33]. However, the molecular mechanism of miR-144-3p remains unclear.

In the present study, we demonstrated that miR-144-3p was a critical oncomiRNA of ccRCC cell proliferation, metastasis and chemoresistance in vitro and promoted tumor growth in vivo. Bioinformatics analyses and dual-luciferase reporter assays indentified ARID1A as a direct target gene of miR-144-3p. Downregulation of ARID1A could promote the invasive capacity and chemoresistance of miR-144-3p in ccRCC. Additionally, we demonstrated that ARID1A was lower in ccRCC tissues in contrast with that in adjacent tissues and did not differ significantly from those in nccRCC or renal hamartoma. Altogether, our results suggest that miR-144-3p acts as an oncogene in ccRCC by direct targeting ARID1A. 


\section{Cellular Physiology Cell Physiol Biochem 2017;43:2420-2433 \begin{tabular}{l|l|l} 
and Biochem 10.1159/000484395 & $\begin{array}{l}\text { (c) } 2017 \text { The Author(s). Published by S. Karger AG, Basel } \\
\text { www.karger.com/cpb }\end{array}$
\end{tabular}}

Xiao et al.: MiR-144-3p Promotes ccRCC by Downregulating ARID1A

\section{Materials and Methods}

\section{Human samples}

Patient samples were obtained from 60 patients with ccRCC, 8 patients with nccRCC and 10 patients with renal hamartoma in the Department of Urology, Union hospital, Tongji Medical College, Wuhan, China. Fresh tumor tissues were frozen in liquid nitrogen after sample collection and stored at $-80{ }^{\circ} \mathrm{C}$ for RNA extraction. Written, informed consent was obtained from each individual and the study was approved by the local ethics committee.

\section{RNA extraction and $q R T-P C R$}

Total RNA of tissues was extracted with the TRizol reagent (Thermo, Massachusetts, USA) according to the manufacturer's instructions. The concentration and purity of the RNA solution were measured by the NanoDrop 2000 spectrophotometer (NanoDrop Technologies, Wilmington, USA). $1 \mu \mathrm{g}$ of enriched tissue or cell RNAs were applied for reverse transcription. Reverse transcription of miRNA was accomplished using the RevertAid First-Strand cDNA Synthesis Kit (Thermo, Massachusetts, USA) according to the manufacturer's instructions, and also with reverse transcript primers from RiboBio (RiboBio, Guangzhou, China). qPCR analysis was performed (LightCycler 480II; Roche, Basel, Switzerland) with the SYBR Green mix (Thermo, Massachusetts, USA) according to the manufacturer's instructions. MiRNAs primers were purchased from RiboBio (RiboBio, Guangzhou, China) and gene primers ARID1A (forward, CTCTATCCAAAACAGGCCGC, reverse, TTTACATCCCCAAGGCCCAT) were purchased from GENEWIZ (GENEWIZ, Suzhou, China). Samples were normalized to GAPDH (forward, 5'-GAGTCAACGGATTTGGTCGT-3'; reverse, 5'-GACAAGCTTCCCGTTCTCAG-3'). Relative expression was calculated using the power formula: $2^{-\Delta \mathrm{Ct}}\left(\Delta \mathrm{Ct}=\mathrm{Ct}_{\text {miR-144-3p }}-\mathrm{Ct}_{\text {normalizer }}\right)$.

\section{Cell culture, infection and transfection}

Human RCC cell line 786-0 was obtained from the American Type Culture Collection and RCC cell line SN12-PM6 was supplied by Dr. I.J. Fidler (MD Anderson Cancer Center). All cells were cultured in DMEM supplemented with $10 \% \mathrm{FBS}$ and $1 \%$ penicillinstreptomycin at $37^{\circ} \mathrm{C}$ in $5 \% \mathrm{CO}$. Lentiviruses containing vector pGCSIL-GFP and pGCSIL-GFP-miR-144 constructed (miR-144-3p, Ant-miR-144-3p) by GENECHEM (GENECHEM, Shanghai, China), Lentiviral vector pLKO.1 or human ARID1A (TRCN0000059090) with shorthairpin RNAs (shRNAs) were used to infect 786-0 or SN12-PM6 cells at an MOI of 10 or 30 respectively. For transfection, SN12-PM6 and 786-0 cells were cultured in 6 well plants and transfected with 100 nM ARID1A siRNA and control siRNA (GenePharma, Shanghai, China) using Lipofectamine 2000 reagents (Thermo Fisher Scientific, Waltham, USA). All steps were executed according to the manufacturer's instructed protocol.

\section{Cell viability, colony formation, cell cycle, migratory, invasion and drug sensitivity assays}

For cell proliferation assay, $4 \times 10^{3}$ cells were plated in 96 -well plates. The cell growth rate was detected using the MTS method according to the manufacturer's instructions. Cell viability was assessed after 24, 48, 72 and 96 hours, respectively. To evaluate the effects of colony formation, 1000 cells were seeded into 6 -well plates. After 14 days, surviving colonies ( $>50$ cells/colony) were counted with $0.05 \%$ crystal violet staining. To analyze cell cycle distribution, cells were cultured to mediums containing 10\% FBS in 6-well plates. Plasma was withdrawn when cells were 70\% confluent. After 24 hours, $10 \%$ FBS was added in the medium and then cultured for an additional 24 hours. Cells were fixed in 70\% ethanol and stained with propidium iodide. DNA content was analyzed through fluorescence-activated cell-sorting (Thermo Fisher Scientific, Waltham, USA). For analysis of migratory and invasion assays, cells were withdrawn of plasma $24 \mathrm{~h}$ for homogenization. Then cells were cultured in 24-well transwell plates with $8 \mathrm{~mm}$ pore polycarbonate membrane inserts (Corning, New York, USA) according to manufacturer's protocol. Then, for the invasion assay, the membrane was coated with the matrigel (Thermo Fisher Scientific, Waltham, USA). After 24 hours of incubation, cells invading the lower surface of the membrane insert were fixed in $100 \%$ methanol, stained with $0.05 \%$ crystal violet, and quantified by counting in 10 random fields. To analyze cell drug sensitivity, $1 \times 10^{4}$ cells were plated in 96 -well plates. After $24 \mathrm{~h}$, cells were treated with various concentrations $(5 \mu \mathrm{M}$, $10 \mu \mathrm{M}, 15 \mu \mathrm{M}, 20 \mu \mathrm{M}$ ) of Sunitinib (Cell Signaling, Massachusetts, USA) and incubated 48h, respectively. Cells livability was detected using MTS method. Three independent experiments were done.

\section{KARGER}




\section{Cellular Physiology Cell Physiol Biochem 2017;43:2420-2433

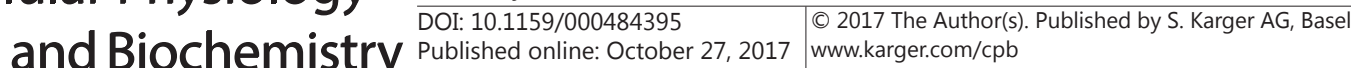

Xiao et al.: MiR-144-3p Promotes ccRCC by Downregulating ARID1A

\section{Tumor formation in nude mice}

For in vivo studies, $1 \times 10^{6}$ SN12-PM6 cells stably expressing miR-144-3p or miR-NC were injected subcutaneously into the flanks of male nude mice (Beijing Hfk Bioscience, Beijing, China) 4-5 weeks of age. Each group contained five mice. Tumor size was measured every 3 days. After 6 weeks, the mice were euthanized and the tumors were harvested. Tumor volumes were calculated as follows: volume $=\left(D \times d^{2}\right) / 2$, where $\mathrm{D}$ was the longest diameter and $\mathrm{d}$ was the shortest diameter. Tumor growth rates were calculated with the specific growth rate (SGR): SGR $=\ln \left(V_{2} / V_{1}\right) /\left(t_{2}-t_{1}\right)$, where $V$ was tumor volume and $t$ was the time of tumor growth as described in [34]. All experiments were approved by the Animal Care and Use Committee of Tongji Medical College of Huazhong University of Science and Technology.

\section{Luciferase assays}

Wild-type and mutant ARID1A 3'UTR reporters were purchased from RiboBio (RiboBio, Guangzhou, China). Tumor cells overexpressing miR-144-3p and miR-NC cultured in 24-well plates were cotransfected with 500ng luciferase reporter with Lipofectamine 2000 reagents (Thermo Fisher Scientific, Waltham, USA). All dual luciferase assays were measured using the Dual Luciferase Assay (Promega, Madison, USA). Normalized renilla-luciferase values were represented relative to the control according to the manufacturer's protocol.

\section{Western blotting and Immunohistochemical staining assays}

For western blotting analyses, the cells were harvested, and all the protein was extracted using RIPA protein lysis buffer (Beyotime Institute of Biotechnology, Haimen, China) with freshly added protease

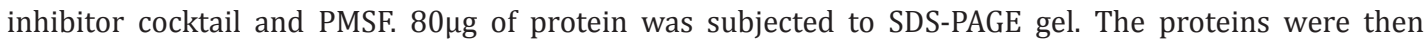
separated by gel electrophoresis and transferred to polyvinylidene fluoride (PVDF) membranes. The membranes were blocked with 5\% non-fat dried skimmed milk and incubated overnight with antibodies against ARID1A (1:1000, Sigma) and $\beta$-actin (1:200, Santa Cruz), and were then washed and incubated in blocking buffer with the appropriate horseradish peroxidase-conjugated secondary antibodies (1:2500, Invitrogen) for 2 hours. The membranes were finally washed before detection. For immunohistochemical staining analyses, briefly, tissue sections $(4 \mu \mathrm{m})$ were dewaxed and rehydrated before performing antigen retrieval. The slides were incubated with anti- ARID1A (1:200, Sigma) overnight at $4^{\circ} \mathrm{C}$, and incubated with an HRP-conjugated secondary antibody for $1 \mathrm{~h}$ at room temperature. DAB was used for color development, and dark brown staining was considered positive. The strength of positivity was semi-quantified by taking into account the percentage of positive cells.

\section{Statistical analysis}

The two-tailed Student's t-test was used for comparisons of two independent groups, apart from the significance of miRNA and proteinlevels in tissues, which was analyzed with the Mann-Whitney test or Wilcoxon test. The Pearson correlation test was performed to examine the correlation between tissue miRNA levels and tissue ARID1A levels. Statistical analysis was done with GraphPad Prism, version 6. P values of $<0.05$ were considered statistically significant.

\section{Results}

MiR-144-3p promoted proliferation and clonogenicity of renal cancer cells

As miR-144-3p was the only upregulated miRNA both in clear cell renal cell carcinomas (ccRCCs) matched with normal kidney tissues and in paired preoperative matched with postoperative plasmas (Table 1), the present study further explores the biological significance of miR-144-3p in renal cancer cell, in which overexpressed miR-144-3p in 786-0 and SN12-PM6 cells with lentivirus carried miR-144-3p and its control miR-NC. The efficacy of infection was confirmed through fluorescence microscopy and efficacy of expressing was verified through qRT-PCR (Fig. 1A, B). MTS analyses showed that SN12-PM6 and 786-0 both proliferated more rapidly than the control when overexpressing miR-144-3p (Fig. 1 C and D). Overexpressing miR-144-3p could fortify both cell lines with the capability of clone formation in vitro (Fig. 4E, F). This was also verified by fluorescence-activated cell-sorting. 
Table 1. List of miRNAs upregulated in cancer tissues vs adjacent normal tissues and preoperative vs postoperative plasmas of ccRCC. Note: Differentially expressed miRNAs were identified by using a filter based on a fold change of 2.0 combined with $p<0.05$ (ANOVA)

\begin{tabular}{lccccc}
\hline $\begin{array}{l}\text { miRNA in cancer/ } \\
\text { normal tissues }\end{array}$ & p value & $\begin{array}{c}\text { Fold } \\
\text { change }\end{array}$ & $\begin{array}{c}\text { miRNA in preoperative } \\
\text { /postoperative plasmas }\end{array}$ & p value & $\begin{array}{c}\text { Fold } \\
\text { change }\end{array}$ \\
\hline hsa-miR-210 & $5.36 \mathrm{E}-04$ & 22.40638 & hsa-miR-144 & $7 \mathrm{E}-05$ & 90.252393 \\
hsa-miR-224 & $4.38 \mathrm{E}-04$ & 14.15244 & hsa-miR-107 & 0.004570 & 14.728802 \\
hsa-miR-34a* & $2.04 \mathrm{E}-05$ & 7.243059 & hsa-miR-19a & 0.009242 & 13.552725 \\
hsa-miR-155 & 0.003397 & 6.138212 & hsa-miR-106b & 0.022127 & 8.3242068 \\
hsa-miR-34b* & $8.22 \mathrm{E}-05$ & 6.100412 & hsa-miR-25 & 0.020859 & 8.2791540 \\
hsa-miR-452 & 0.006482 & 5.675232 & hsa-miR-17 & 0.029819 & 5.7656087 \\
hsa-miR-34a & $2.46 \mathrm{E}-05$ & 5.334161 & hsa-miR-20a & 0.047278 & 4.2103129 \\
hsa-miR -142-5p & 0.009996 & 4.448587 & hsa-miR-451 & 0.007255 & 3.2902160 \\
hsa-miR-1271 & 0.005688 & 3.984044 & hsa-miR-19b & 0.030042 & 2.5658867 \\
hsa-miR-144 & 0.019844 & 3.84862 & & & \\
hsa-miR-21* & 0.012136 & 3.45346 & & & \\
hsa-miR-342-5p & $1.65 \mathrm{E}-04$ & 3.427933 & & & \\
hsa-miR-21 & 0.002899 & 3.281815 & & & \\
hsa-miR-16-2* & $7.16 \mathrm{E}-04$ & 2.733205 & & & \\
hsa-miR-143* & 0.001727 & 2.733057 & & & \\
hsa-miR-142-3p & 0.043484 & 2.657934 & & & \\
hsa-miR-181c & 0.008708 & 2.542275 & & & \\
\hline
\end{tabular}

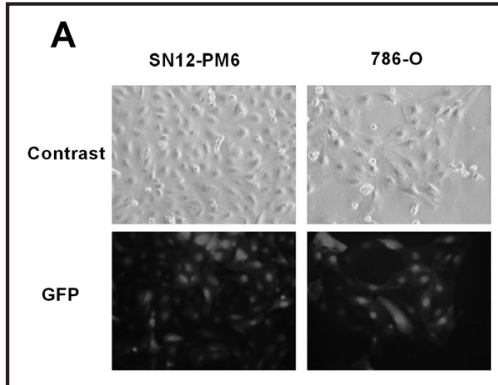

B

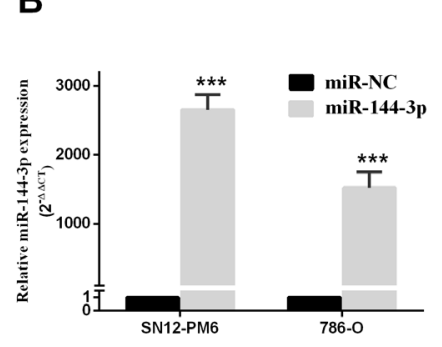

E

C

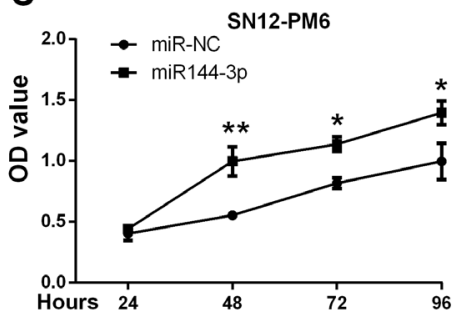

G

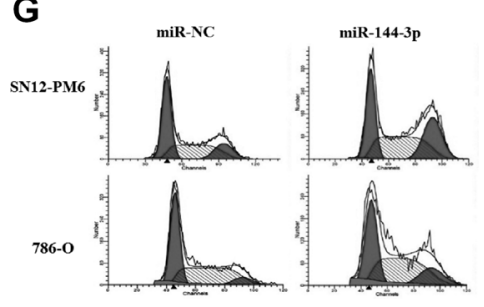

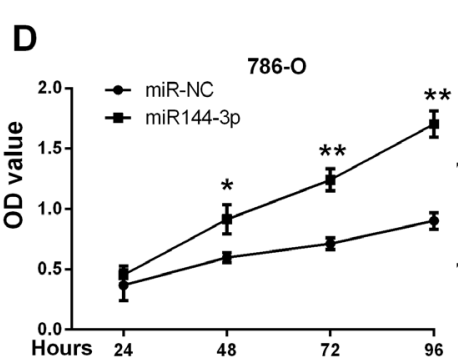

H

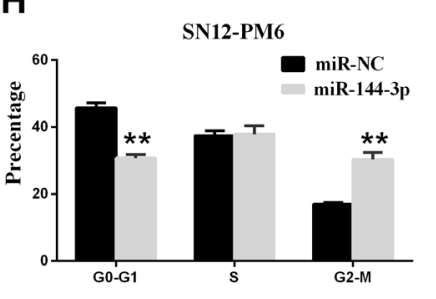

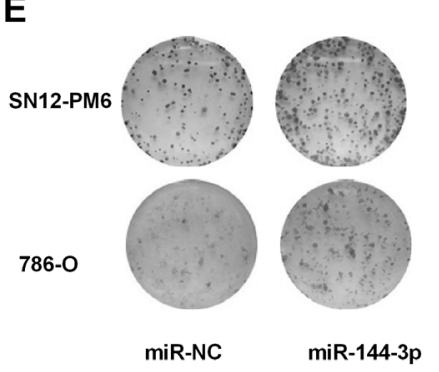

$\mathbf{F}$

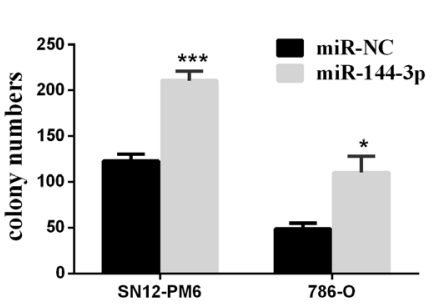

I

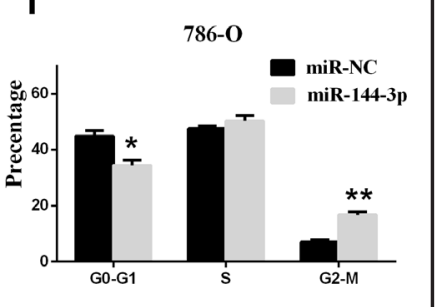

Fig. 1. Mir-144-3p promoted RCC cell growth and proliferation in vitro. (A) and (B) Infective effects and relative expression of miR-144-3p in SN12-PM6 and 786-O cell lines with lentivirus infection were assessed by fluorescence microscope and qRT-PCR respectively. Empty vector (miR-NC) infected RCC cells used as control. (C) and (D) Effect of miR-144-3p on cell proliferation was measured by MTS assay in miR-144-3poverexpressing SN12-PM6 and 786-0. (E) and (F) Representative picture of colony formation assay in miR144-3p-overexpressing SN12-PM6 and 786-O cells and quantitative analyses of colony numbers. (G), (H) and (I) Representative charts for cell-cycle distribution in both ccRCC cells and cell-cycle statistical analysis of SN12-PM6 and 786- 0 cells. ${ }^{*} \mathrm{P}<0.05$; ${ }^{* *} \mathrm{P}<0.01$; ${ }^{* * *} \mathrm{P}<0.001$. 
Fig. 2. Mir-144-3p promoted 786-0 and SN12-PM6 migration, invasion and chemoresistance. (A) and (B) Representative pictures showed the capability of migration or invasion in miR-144-3poverexpressing SN12-PM6 and 786-0 cells versus corresponding control cells. (C) and (D) Transwell statistical analysis of SN12-PM6 and 786-0; Columns, mean of at least ten random fields. Original magnification was $\times 400$. (E) and (F) The capability of resistant to Sunitinib in miR-144-3p-overexpressing

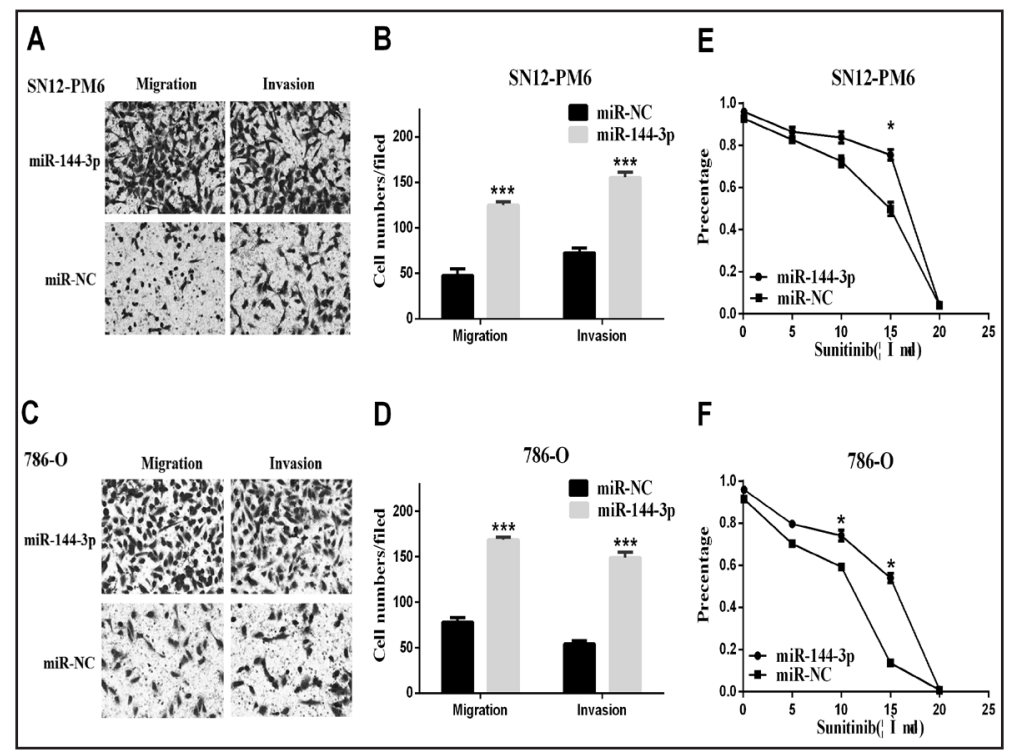

SN12-PM6 and 786-0 cells was measured by MTS assay according diverse concentrations $(5 \mu \mathrm{M}, 10 \mu \mathrm{M}$, $15 \mu \mathrm{M}, 20 \mu \mathrm{M}$ ) in $48 \mathrm{~h}$, respectively. ${ }^{*} \mathrm{P}<0.05$; ${ }^{* * *} \mathrm{P}<0.001$.

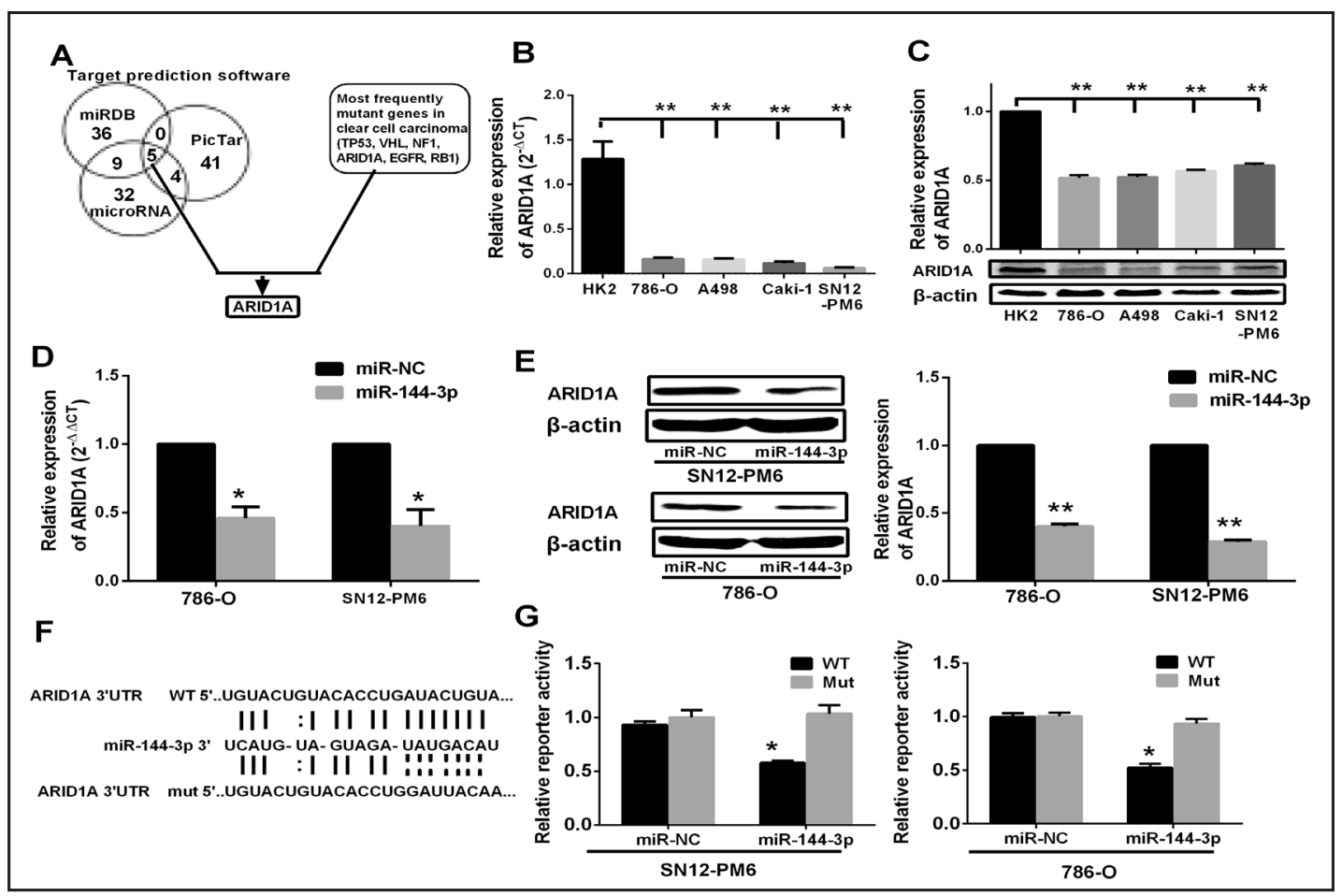

Fig. 3. ARID1A was a direct target gene of miR-144-3p in ccRCC. (A) Bioinformatic prediction of candidated mRNAs targeting of miR-144-3p. (B) and (C) qRT-PCR and western blotting analysis of ARID1A expression in normal human kidney cell line HK2 and 4 cultured renal cell carcinoma lines. (D) and (E) MiR-144-3p downregulated ARID1A at the mRNA and protein levels. qRT-PCR and western blotting analysis of ARID1A expression in miR-144-3p-overexpressing SN12-PM6 and 786-0 cells versus corresponding control cells. (F) The predicted binding sites for miR-144-3p in ARID1A 3'UTR. The red nucleotides are the seed-pairing target sites of miR-144-3p. (G) Luciferase reporter assays showing about $50 \%$ decreased reporter activity after transfection of wild-type ARID1A 3'UTR reporter construct in the SN12-PM6 and 786-0 cells overexpressing miR-144-3p. In all panels, bar graphs represent mean \pm SEM of three independent experiments. The statistical significance of all tests was accepted for $* \mathrm{P}<0.05$; ${ }^{* *} \mathrm{P}<0.01$. 


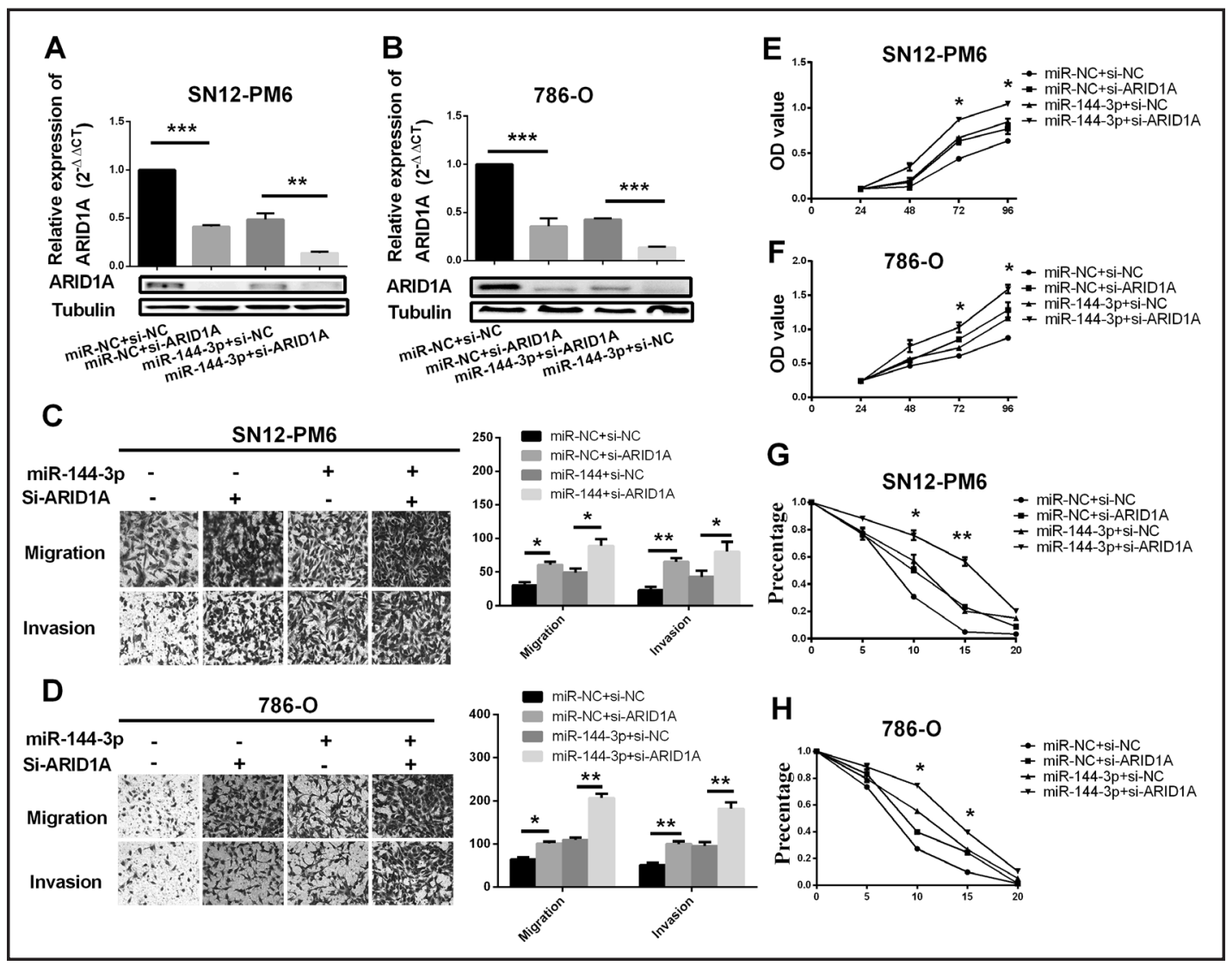

Fig. 4. Knockdown of ARID1A in ccRCC cells significantly promoted cell growth, increased cell migration and invasion and chemoresistance. (A) and (B) The mRNA and protein levels of ARID1A in SN12-PM6, 7860 and miR-144-3p-overexpressing cells were assessed by qRT-PCR and western blot analysis, respectively. Cells were transfected with $100 \mathrm{nmol} / \mathrm{L}$ ARID1A siRNA versus nonspecific control for 48 hours before detection. Tubulin served as internal controls for protein loading. (C) and (D) Transwell migration and invasion assays of SN12-PM6 and 786-0 cells were performed after transfection with ARID1A siRNA (si-ARID1A) versus control (si-NC) for 48 hours. (E) and (F) MTS assay cell proliferation of SN12-PM6, 786-0 and miR144-3p-overexpressing cells were performed after transfection with ARID1A siRNA (si-ARID1A) versus control (si-NC) for 48 hours. (G) and (H) MTS assay the capability of resistant to Sunitinib in SN12-PM6, 786-0 and miR-144-3p-overexpressing cells by siRNA against AIRD1A or negative control. The statistical significance of all tests was accepted for $* \mathrm{P}<0.05$; ${ }^{*} \mathrm{P}<0.01$.

When overexpressing miR-144-3p, G0-G1 phase cells obviously decreased, while G2-GM phase cells greatly increased (Fig. 1G, H, I)

MiR-144-3p enhanced migration, invasion and resistant to Sunitinib capability of renal cancer cells

Given the functional annotation term of miR-144-3p accelerate renal cancer cell proliferation and clone formation, we next assessed the potential role of miR-144-3p in renal cancer cell migration and invasion through the transwell assay. The result showed that miR144-3p overexpression was able to significantly heighten the capability of migration and invasion in SN12-PM6 and 786-0 cells (Fig. 2 A, B, C, D). Furthermore, we found the cell lines overexpressing miR-144-3p could enhance the strength of resistance to Sunitinib. As shown in (Fig. 2 E, F), SN12-PM6 and 786-O with overexpressed miR-144-3p were distinctly tougher than the control in $15 \mu \mathrm{mol}$ Sunitinib when both were cultured for $48 \mathrm{~h}$. 
Fig. 5. ARID1A was downregulated and had a negative correlation with miR-144$3 p$ overexpression in ccRCC patients. (A), (B) and (C) qRT-PCR analysis expression of ARID1A mRNA in 60 ccRCC, 8 nccRCC and 10 renal hamartoma patients, ARID1A was downregulated in ccRCC in matched normal kidney tissues patients $(\mathrm{P}<0.0001)$ but had no difference in nccRCC ( $P=0.5469)$ or renal hamartoma patients ( $\mathrm{P}=0.7695)$. (D) The mRNA expression of ARID1A was negatively correlated with miR144-3p expression in ccRCC tissues $(\mathrm{R}=$ -0.396, $P=0.002$ ). (E) The mRNA expression of ARID1A was lower with high miR144-3p expression in
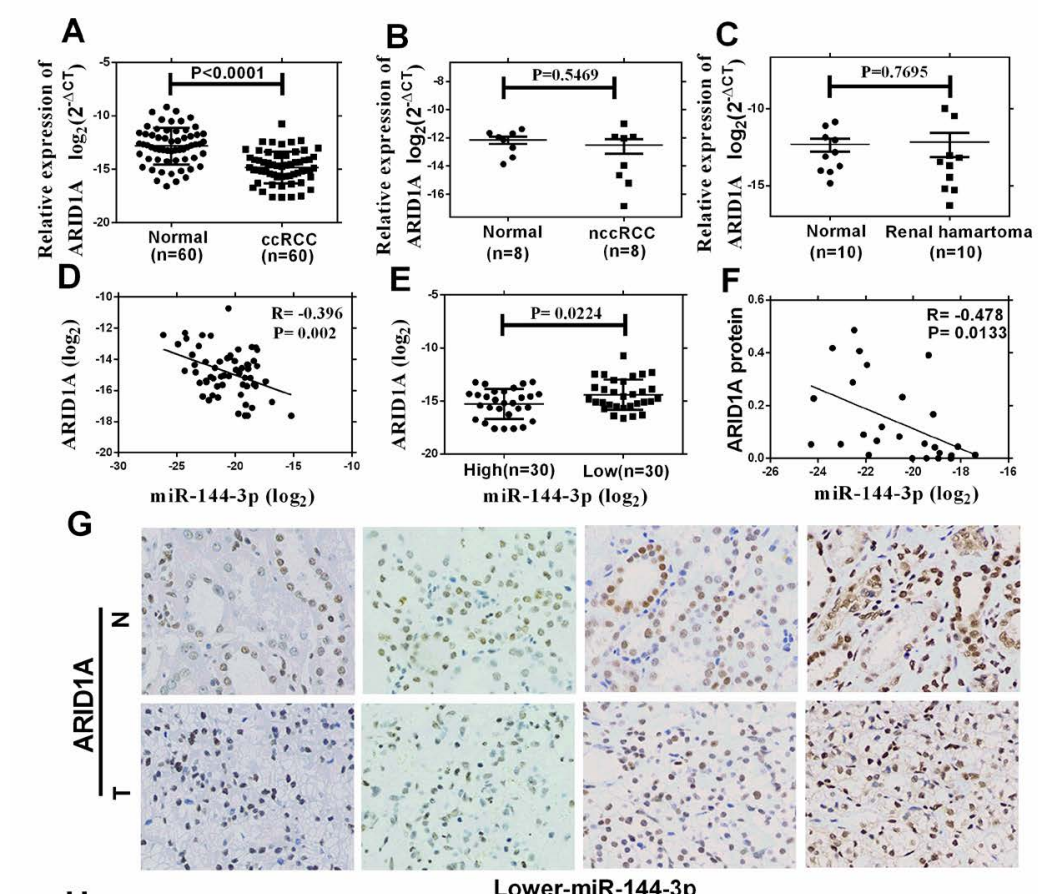

$\mathrm{H}$

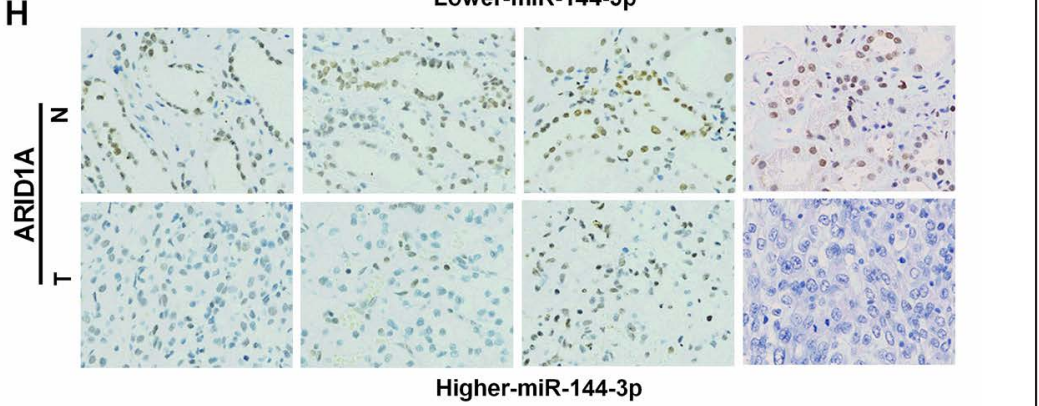
ccRCC tissues when divided into 2 groups ( $\mathrm{P}=0.0224)$. (F) The protein expression of ARID1A was negatively correlated with miR-144-3p expression in 26 ccRCC tissues $(R=-0.496, P=0.03)$. $(G)$ and $(H)$ The protein expression of ARID1A by IHC in ccRCC samples (T) and in matched normal kidney tissues (N) of two group.

\section{ARID1A was a direct downstream target of miR-144-3p}

The above data implies that miR-144-3p is a key oncomiRNA for ccRCC. In order to identify the potential target of miR-144-3p, computational prediction software, including miRDB, PicTar and microRNA were used to predict possible molecules. We choose the top 50 predict molecules in each group and identified a total of 5 common molecules. To narrow the field of prospective candidates, we further analyzed the potential candidates by overlapping this result with most mutation molecules in ccRCC $[7,10]$, and gave evidence that ARID1A could be a potential target (Fig. 3A). We then performed an analysis of ARID1A expression in immortalized human renal tubule epithelial cell line HK-2 and indicated renal carcinoma cell lines, which were expressed at lower levels in a panel of 4 human renal carcinoma cell lines, with Real-time PCR and western blotting (Fig. 3B, C). Upregulation of miR-144-3p with lentivirus led to a marked decrease in ARID1A mRNA and protein levels and much lower than control levels, according to qRT-PCR and western blot results (Fig. 3D, E). Next, we determined the effect of miR-144-3p on the $3^{\prime}$-UTR of ARID1A in renal cancer cells using the luciferase reporter assay. As shown (Fig. 3F, G), miR-144-3p overexpression significantly 


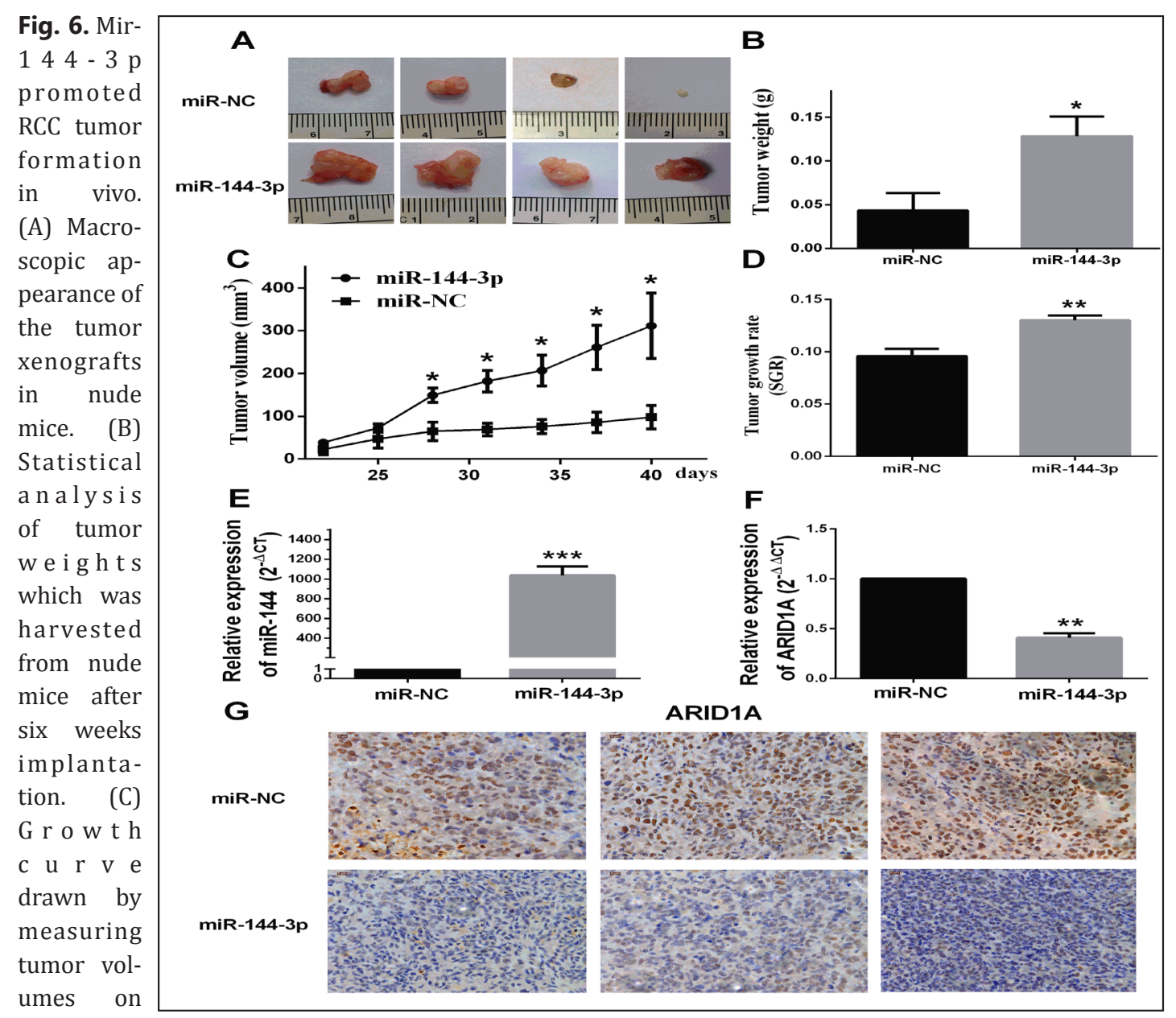

the indicat-

ed days. Error bars represent the SEM. (D) Tumor growth rates were calculated by the specific growth rate (SGR): $S G R=\ln \left(V_{2} / V_{1}\right) /\left(t_{2}-t_{1}\right), V=$ tumor volume, $t=$ days. ${ }^{*} \mathrm{P}<0.05$; ** $\mathrm{P}<0.01$. (E), (F) and (G) miR-144-3p significantly decreased the abundance of ARID1A and in tumors as compared to controls with qRT-PCR and IHC.

repressed the activity of wild-type reporters but not mutant 3' UTRs of ARID1A. Collectively, these results indicated that ARID1A was a direct target of miR-144-3p.

\section{Inhibiting ARID1A significantly enhanced the proliferation and metastasis of renal cancer} cells

We then further investigated the function of ARID1A in renal cancer cells by inhibiting ARID1A expression with siRNA in SN12-PM6 and 786-O and lentivirus miR-144-3p cells. qRT-PCR and Western blotting were used to analyze the mRNA and the protein level of ARID1A (Fig. 4A, B). ARID1A silencing could significantly enhance the capability of migration and invasion in lentivirus miR-144-3p cells using transwell assays (Fig. 4C, D). Moreover, The MTS analyses revealed that ARID1A-depleted cells had more powerful proliferated activity compared with control cells (Fig. 4E and F). Furthermore, inhibiting ARID1A could greatly increase resistance to Sunitinibi (Fig. 4G and H).

\section{ARID1A was significantly decreased in ccRCC}

We detected the expression of ARID1A in 60 patients with ccRCC using qRT-PCT and found that the mRNA expression of ARID1A significantly decreased in ccRCC tissues compared with adjacent normal tissues (Fig. 5A), but this difference did not differ from that

\section{KARGER}


Fig. 7. ARID1A reversed the function of miR-144-3p in the tumor growth of SN12-PM6 cells in vivo. (A) The levels of miR-144 and ARID1A mRNA in SN12-PM6 cells. (B) the levels of ARID1A protein Cells in SN12-PM6 cells. (C) Macroscopic appearance of the tumor xenografts in nude mice. (D) Growth curve drawn by measuring tumor volumes on the indicated days. Error bars represent the SEM. (E) Statistical analysis of tumor weights which was harvested from nude mice. (F) Tumor growth rates were calculated by the specific growth rate (SGR): $S G R=\ln \left(V_{2} / V_{1}\right) /\left(t_{2}-t_{1}\right), V=$ tumor volume, $\mathrm{t}=$ days. ${ }^{*} \mathrm{P}<0.05$; ** $\mathrm{P}<0.01$. *** $\mathrm{P}<0.001$.

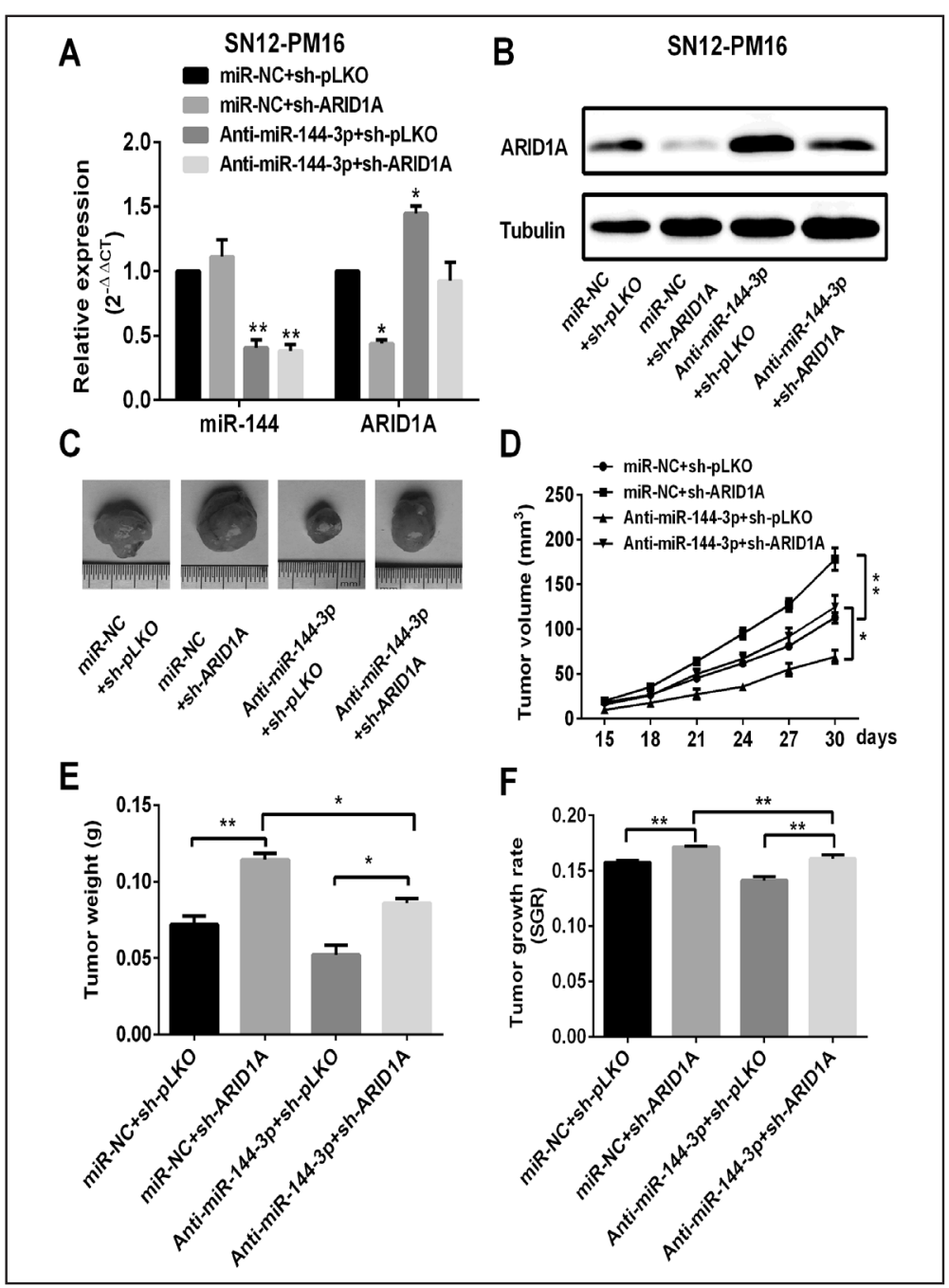

between nccRCC tissues or renal hamartoma tissues compared with adjacent normal tissues (Fig. 5B, C). We then found a significant negative correlation between ARD1A and miR-144$3 p$ in mRNA expression ( $R=-0.396, P=0.002$ ) (Fig. 5D). Specifically, higher miR-144 levels were associated with lower ARID1A mRNA expression, and vice versa (Fig. 5E). A similar result was obtained in protein level using immunohistochemistry (IHC) analysis in 26 patients paired ccRCC tissues with normal tissues (Fig. 5G, H), statistical analysis revealed ARID1A protein levels were negatively correlated with miR-144-3p levels $(R=-0.478, P=$ 0.0133 ) in ccRCC tissues (Fig. 5F). Collectively, these findings indicate that ARID1A was a direct and functional target of miR-144-3p.

\section{MiR-144-3p promoted tumor formation in vivo}

To demonstrate that miR-144-3p could promote ccRCC tumor growth by targeting ARID1A in vivo, tumor formation assay was performed by subcutaneous injection of SN12PM6 cells stably expressing miR-144-3p or miR-NC into the flank of male nude mice. All 5 mice injected with miR-144-3p cells formatted tumors, while 4/5 mice injected with miRNC cells developed tumors. The size of subcutaneous tumors originating from miR-144-3ptransduced SN12-PM6 cells was dramatically larger than that of miR-NC-transduced cells (Fig. 6A). The average tumor volume, tumor weight and tumor growth rate of miR-144-3ptreated group were all significantly increased compared with control (Fig. 6 B, C, D). The expression of miR-144-3p in xenografts was higher in miR-144-3p-transduced cells than 


\section{Cellular Physiology Cell Physiol Biochem 2017;43:2420-2433

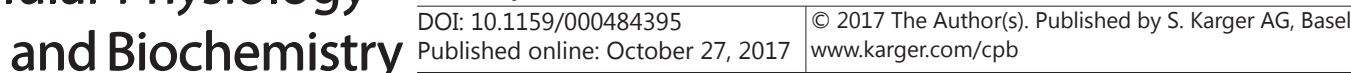 \\ Xiao et al.: MiR-144-3p Promotes ccRCC by Downregulating ARID1A}

that in miR-NC-transduced cells (Fig. 6E). Moreover, miR-144-3p significantly decreased the abundance of ARID1A in tumors as compared to controls with qRT-PCR and IHC (Fig. 6F, $\mathrm{G})$. We also found that ARID1A reversed the function of miR-144-3p in the tumor growth of SN12-PM6 cells in vivo (Fig. 7). Taken together, the data shows that miR-144-3p has potent protumor activity for ccRCC by targeting ARID1A to promote cancer proliferation, metastasis in vitro and in vivo.

\section{Discussion}

MiR-144-3p is an important miRNA that dysregulates in many tumors. Many studies have revealed that miR-144-3p downregulated in non-small-cell lung cancer, bladder cancer, and hepatocellular cancer as an anti-tumor miRNA [35-37]. However, other studies indicate that miR-144-3p increased in nasopharyngeal carcinoma as an oncomiRNA [38]. We previously found that miR-144-3p was the only one upregulated miRNA both in clear cell renal cell carcinoma (ccRCC) tissue samples matched with normal kidney tissues and in paired preoperative matched with postoperative plasmas [32, 33]. However, the mean level of miR-144-3p was downregulated in non-clear-renal-cell-carcinoma tissues compared with adjacent normal tissues, but had no statistical significance. In this study, we stably overexpressed miR-144-3p in 786-O and SN12-PM6 with lentiviruses and found that miR144-3p overexpression could promote proliferation and motility in ccRCC cells. Thus, we identified miR-144-3p as a key onco-miRNA in renal cancer cells. Moreover, we domesticated the expression of miR-144-3p in renal cancer cells was increased compared with normal renal cells which revealed the carcinogenesis of miR-144-3p with qRT-PCR and western blotting. However, we found the level of miR-144-3p in cells, no matter tumorous or normal, was significantly lower than that of renal tissue. We suspect the reason for this phenomenon is the diversity of metabolic components, especially lipid components. ccRCC tissues contain more lipid components, especially cholesteryl ester, than nccRCC tissues and renal cell lines, which were regulated by miR-144-3p according to several reports [39-41]. However, the exact mechanism of regulation of cholesteryl ester by miR-144-3p in ccRCC is unclear, and further study is necessary needed.

MiRNAs participate in many biological activities, which comprise the majority of drug resistance in cancer. It had been reported that down-regulation miR-141 could enhanced the resistance of Sunitinib which used as first-line therapy for mRCC through induction of epithelial-to-mesenchymal transition (EMT) and hypoxia resistance [42, 43]. Another member of miR-200 family, miR-200c, was also reported to be involved in EMT and could sensitize ccRCC cells to sorafenib or imatinib partly by targeting HO-1 [44]. Furthermore, miR-124 was demonstrated to have up-regulation in chemo-resistant RCC cells by targeting P-glycoprotein [45]. All this data identified miRNAs as the key regulators of drug sensitivity and progression in renal cell carcinomas. Our study found overexpression of miR-144$3 p$ could enhance the chemo-resistance in renal cell cells, which provided another novel potential therapeutical target in cancerous chemotherapy.

ARID1A is a key member of the SWI/SNF chromatin-modeling complex, which functions as a negative regulator in cell cycle, apoptosis and tumorigenicity [46]. It has been demonstrated that ARID1A down-regulated p53-related genes [47] and Myc protein. Additionally, ARID1A protein overexpression was reported to significantly increase the sensitivity of cancer cells towards PI3K- and AKT-inhibitors, which suggests a dependency of ARID1A-mutated tumors on activating the mechanism of the PI3K/AKT-pathway [48]. In human tumors, ARID1A was found mutated or missing frequently in carcinomas of the breast, ovarian, lung and kidney in several recent reports [49-51]. Comprehensive molecular studies revealed that ARID1A mutations exist in 3\% of ccRCC [52]. But recent articles show that $12 \%$ mRNA mutation in genomic alterations (GAs) [10] and 51\% protein loss of ARID1A [13] in ccRCC. It indicated that someone down-regulated ARID1A in translation, yet did not only interfere on the genetic level. Recently, ARID1A mRNA and BAF250a have been suggested 
as new potential prognostic markers in ccRCC. Meanwhile, we found ARID1A mRNA and protein are both negatively correlated with ccRCC miR-144-3p expression ARID1A. This suggests that the expression level of miR-144-3p in ccRCC tissues also might be a prognostic marker of ccRCC, but this requires a long-term study to confirm. Furthermore, we identified ARID1A did not significantly differ between 8 nccRCCs and 10 renal hamartoma patients, which corresponds to the expression of miR-144-3p in nccRCC tissues.

Our study indicated that miR-144-3p specifically down-regulate ARID1A and took its effect on ccRCC. It is the first report that identified ARID1A as a potential target gene of miR-144-3p in ccRCC. However, it might be limited in our study and further study may be essential for our future studies.

\section{Conclusion}

Collectively, we identified that miR-144-3p acts as a critical oncomiRNA in renal cancer cells, which play an important role in the malignant progression of RCC cells by directly targeting ARID1A. Our study provides a novel mechanism in progression and Sunitinib resistance of ccRCC, and reveals that miR-144-3p might be a potential target for ccRCC therapy in future.

\section{Acknowledgements}

This study was supported by grants from the National Natural Scientific Foundation of China (81372760, 81672528 and 81773282). We thank Ke Chen and Qingyang Zhang for assistance with manuscript editing.

\section{Disclosure Statement}

No disclosure statement exists in the submission of this manuscript

\section{References}

1 Siegel RL, Miller KD, Jemal A: Cancer Statistics, 2017. CA Cancer J Clin 2017;67:7-30.

2 Chen W, Zheng R, Baade PD, Zhang S, Zeng H, Bray F, Jemal A, Yu XQ He J: Cancer statistics in China, 2015.

CA Cancer J Clin 2016;66:115-132.

3 Linehan WM: The genetic basis of kidney cancer: implications for management and use of targeted therapeutic approaches. Eur Urol 2012;61:896-898.

4 Linehan WM, Srinivasan R, Schmidt LS: The genetic basis of kidney cancer: a metabolic disease. Nat Rev Urol 2010;7:277-285.

5 Rini BI, Campbell SC, Escudier B: Renal cell carcinoma. Lancet 2009;373:1119-1132.

6 Gong J, Maia MC, Dizman N, Govindarajan A, Pal SK: Metastasis in renal cell carcinoma: Biology and implications for therapy. Asian Journal Of Urology 2016;3:286-292.

-7 Cancer Genome Atlas Research N: Comprehensive molecular characterization of clear cell renal cell carcinoma. Nature 2013;499:43-49.

-8 Cornejo KM, Lu M, Yang P, Wu S, Cai C, Zhong WD, Olumi A, Young RH, Wu CL: Succinate dehydrogenase B: a new prognostic biomarker in clear cell renal cell carcinoma. Hum Pathol 2015;46:820-826.

-9 Yang L, Zhao Z, Zhao S, Chen C, Cong X, Li Z, Ren M: The Clinicopathological Significance of Epigenetic Silencing of VHL Promoter and Renal Cell Carcinoma: A Meta-Analysis. Cell Physiol Biochem 2016;40:14651472.

10 Pal SK, Sonpavde G, Agarwal N, Vogelzang NJ, Srinivas S, Haas NB, Signoretti S, McGregor BA, Jones J, Lanman RB, Banks KC, Choueiri TK: Evolution of Circulating Tumor DNA Profile from First-line to Subsequent Therapy in Metastatic Renal Cell Carcinoma. Eur Urol 2017;72:557-564.

11 Ma W, Tao L, Wang X, Liu Q, Zhang W, Li Q, He C, Xue D, Zhang J, Liu C: Sorafenib Inhibits Renal Fibrosis Induced by Unilateral Ureteral Obstruction via Inhibition of Macrophage Infiltration. Cell Physiol Biochem 2016;39:1837-1849. 


\section{Cellular Physiology Cell Physiol Biochem 2017;43:2420-2433

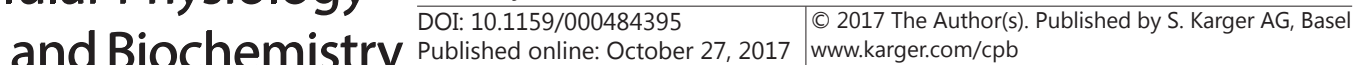

Xiao et al.: MiR-144-3p Promotes ccRCC by Downregulating ARID1A

12 Park JH, Lee C, Suh JH, Chae JY, Kim HW, Moon KC: Decreased ARID1A expression correlates with poor prognosis of clear cell renal cell carcinoma. Hum Pathol 2015;46:454-460.

13 Jiang W, Dulaimi E, Devarajan K, Parsons T, Wang Q O’Neill R, Solomides C, Peiper SC, Testa JR, Uzzo R, Yang $\mathrm{H}$ : Intratumoral heterogeneity analysis reveals hidden associations between protein expression losses and patient survival in clear cell renal cell carcinoma. Oncotarget 2017;8:37423-37434.

14 Guan B, Rahmanto YS, Wu RC, Wang Y, Wang Z, Wang TL, Shih Ie M: Roles of deletion of Arid1a, a tumor suppressor, in mouse ovarian tumorigenesis. J Natl Cancer Inst 2014;106:

-15 Tan Q Cui J, Huang J, Ding Z, Lin H, Niu X, Li Z, Wang G, Luo Q Lu S: Genomic Alteration During Metastasis of Lung Adenocarcinoma. Cell Physiol Biochem 2016;38:469-486.

16 Heestand GM, Kurzrock R: Molecular landscape of pancreatic cancer: implications for current clinical trials. Oncotarget 2015;6:4553-4561.

$\checkmark 17$ Rao CV, Asch AS, Yamada HY: Frequently mutated genes/pathways and genomic instability as prevention targets in liver cancer. Carcinogenesis 2017;38:2-11.

18 Lee RC, Feinbaum RL, Ambros V: The C. elegans heterochronic gene lin-4 encodes small RNAs with antisense complementarity to lin-14. Cell 1993;75:843-854.

19 Hiyoshi Y, Kamohara H, Karashima R, Sato N, Imamura Y, Nagai Y, Yoshida N, Toyama E, Hayashi N, Watanabe M, Baba H: MicroRNA-21 regulates the proliferation and invasion in esophageal squamous cell carcinoma. Clin Cancer Res 2009;15:1915-1922.

20 Gao Y, Ma X, Yao Y, Li H, Fan Y, Zhang Y, Zhao C, Wang L, Ma M, Lei Z, Zhang X: miR-155 regulates the proliferation and invasion of clear cell renal cell carcinoma cells by targeting E2F2. Oncotarget 2016;7:20324-20337.

21 Chen H, Liu C, Jiang H, Gao Y, Xu M, Wang J, Liu S, Fu Y, Sun X, Xu J, Zhang J, Dai L: Regulatory Role of miRNA-375 in Expression of BMP15/GDF9 Receptors and its Effect on Proliferation and Apoptosis of Bovine Cumulus Cells. Cell Physiol Biochem 2017;41:439-450.

-22 Ma L, Teruya-Feldstein J, Weinberg RA: Tumour invasion and metastasis initiated by microRNA-10b in breast cancer. Nature 2007;449:682-688.

23 Zhou Q Zheng X, Chen L, Xu B, Yang X, Jiang J, Wu C: Smad2/3/4 Pathway Contributes to TGF-beta-Induced MiRNA-181b Expression to Promote Gastric Cancer Metastasis by Targeting Timp3. Cell Physiol Biochem 2016;39:453-466.

24 Huang X, Taeb S, Jahangiri S, Emmenegger U, Tran E, Bruce J, Mesci A, Korpela E, Vesprini D, Wong CS, Bristow RG, Liu FF, Liu SK: miRNA-95 mediates radioresistance in tumors by targeting the sphingolipid phosphatase SGPP1. Cancer Res 2013;73:6972-6986.

25 Chen J, Xu Y, Tao L, Pan Y, Zhang K, Wang R, Chen LB, Chu X: MiRNA-26a Contributes to the Acquisition of Malignant Behaviors of Doctaxel-Resistant Lung Adenocarcinoma Cells through Targeting EZH2. Cell Physiol Biochem 2017;41:583-597.

-26 Lu YC, Cheng AJ, Lee LY, You GR, Li YL, Chen HY, Chang JT: MiR-520b as a novel molecular target for suppressing stemness phenotype of head-neck cancer by inhibiting CD44. Sci Rep 2017;7:2042.

27 Rupaimoole R, Slack FJ: MicroRNA therapeutics: towards a new era for the management of cancer and other diseases. Nat Rev Drug Discov 2017;16:203-222.

28 Bartel DP: MicroRNAs: genomics, biogenesis, mechanism, and function. Cell 2004;116:281-297. Ha M, Kim VN: Regulation of microRNA biogenesis. Nat Rev Mol Cell Biol 2014;15:509-524.

30 Mitchell PS, Parkin RK, Kroh EM, Fritz BR, Wyman SK, Pogosova-Agadjanyan EL, Peterson A, Noteboom J, O’Briant KC, Allen A, Lin DW, Urban N, Drescher CW, Knudsen BS, Stirewalt DL, Gentleman R, Vessella RL, Nelson PS, Martin DB, Tewari M: Circulating microRNAs as stable blood-based markers for cancer detection. Proc Natl Acad Sci U S A 2008;105:10513-10518.

-31 Yang Y, Peng W, Tang T, Xia L, Wang XD, Duan BF, Shu Y: MicroRNAs as promising biomarkers for tumorstaging: evaluation of MiR21 MiR155 MiR29a and MiR92a in predicting tumor stage of rectal cancer. Asian Pac J Cancer Prev 2014;15:5175-5180.

32 Lou N, Ruan AM, Qiu B, Bao L, Xu YC, Zhao Y, Sun RL, Zhang ST, Xu GH, Ruan HL, Yuan CF, Han WW, Shi HC, Yang HM, Zhang XP: miR-144-3p as a novel plasma diagnostic biomarker for clear cell renal cell carcinoma. Urol Oncol 2017;35:36 e37-36 e14.

-33 Chen X, Wang X, Ruan A, Han W, Zhao Y, Lu X, Xiao P, Shi H, Wang R, Chen L, Chen S, Du Q, Yang H, Zhang $\mathrm{X}$ : miR-141 is a key regulator of renal cell carcinoma proliferation and metastasis by controlling EphA2 expression. Clin Cancer Res 2014;20:2617-2630. 


\section{Cellular Physiology Cell Physiol Biochem 2017;43:2420-2433

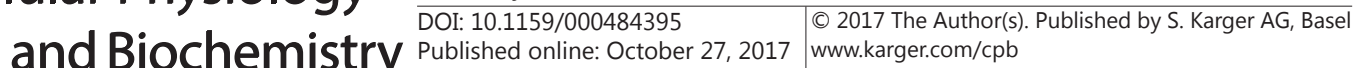

Xiao et al.: MiR-144-3p Promotes ccRCC by Downregulating ARID1A

34 Tian X, Dai S, Sun J, Jiang S, Sui C, Meng F, Li Y, Fu L, Jiang T, Wang Y, Su J, Jiang Y: Inhibition of MDM2 Re-Sensitizes Rapamycin Resistant Renal Cancer Cells via the Activation of p53. Cell Physiol Biochem 2016;39:2088-2098.

35 Zha W, Cao L, Shen Y, Huang M: Roles of Mir-144-ZFX pathway in growth regulation of non-small-cell lung cancer. PLoS One 2013;8:e74175.

-36 Guo Y, Ying L, Tian Y, Yang P, Zhu Y, Wang Z, Qiu F, Lin J: miR-144 downregulation increases bladder cancer cell proliferation by targeting EZH2 and regulating Wnt signaling. FEBS J 2013;280:4531-4538.

-37 Ma Y, She XG, Ming YZ, Wan QQ Ye QF: MicroRNA144 suppresses tumorigenesis of hepatocellular carcinoma by targeting AKT3. Mol Med Rep 2015;11:1378-1383.

38 Zhang LY, Ho-Fun Lee V, Wong AM, Kwong DL, Zhu YH, Dong SS, Kong KL, Chen J, Tsao SW, Guan XY, Fu L: MicroRNA-144 promotes cell proliferation, migration and invasion in nasopharyngeal carcinoma through repression of PTEN. Carcinogenesis 2013;34:454-463.

-39 de Aguiar Vallim TQ, Tarling EJ, Kim T, Civelek M, Baldan A, Esau C, Edwards PA: MicroRNA-144 regulates hepatic ATP binding cassette transporter A1 and plasma high-density lipoprotein after activation of the nuclear receptor farnesoid X receptor. Circ Res 2013;112:1602-1612.

40 He J, Tian Y, Zhao Y, Liu Y, Tao Z, Li G, Niu D, Lu L, Lu Y: MiR-144 affects fatty acid composition by regulating ELOVL6 expression in duck hepatocytes. Cell Biol Int 2017;41:691-696.

41 He J, Wang W, Lu L, Tian Y, Niu D, Ren J, Dong L, Sun S, Zhao Y, Chen L, Shen J, Li X: Analysis of miRNAs and their target genes associated with lipid metabolism in duck liver. Sci Rep 2016;6:27418.

-42 Berkers J, Govaere O, Wolter P, Beuselinck B, Schoffski P, van Kempen LC, Albersen M, Van den Oord J, Roskams T, Swinnen J, Joniau S, Van Poppel H, Lerut E: A possible role for microRNA-141 down-regulation in sunitinib resistant metastatic clear cell renal cell carcinoma through induction of epithelial-tomesenchymal transition and hypoxia resistance. J Urol 2013;189:1930-1938.

-43 Pal SK, Bergerot P, Figlin RA: Renal cell carcinoma: An update for the practicing urologist. Asian Journal of Urology 2015;2:19-25.

44 Gao C, Peng FH, Peng LK: MiR-200c sensitizes clear-cell renal cell carcinoma cells to sorafenib and imatinib by targeting heme oxygenase-1. Neoplasma 2014;61:680-689.

45 Long QZ, Du YF, Liu XG, Li X, He DL: miR-124 represses FZD5 to attenuate P-glycoprotein-mediated chemoresistance in renal cell carcinoma. Tumour Biol 2015;36:7017-7026.

46 Yan HB, Wang XF, Zhang Q, Tang ZQ, Jiang YH, Fan HZ, Sun YH, Yang PY, Liu F: Reduced expression of the chromatin remodeling gene ARID1A enhances gastric cancer cell migration and invasion via downregulation of E-cadherin transcription. Carcinogenesis 2014;35:867-876.

47 Guan B, Wang TL, Shih Ie M: ARID1A, a factor that promotes formation of SWI/SNF-mediated chromatin remodeling, is a tumor suppressor in gynecologic cancers. Cancer Res 2011;71:6718-6727.

48 Liang H, Cheung LW, Li J, Ju Z, Yu S, Stemke-Hale K, Dogruluk T, Lu Y, Liu X, Gu C, Guo W, Scherer SE, Carter H, Westin SN, Dyer MD, Verhaak RG, Zhang F, Karchin R, Liu CG, Lu KH, Broaddus RR, Scott KL, Hennessy BT, Mills GB: Whole-exome sequencing combined with functional genomics reveals novel candidate driver cancer genes in endometrial cancer. Genome Res 2012;22:2120-2129.

49 Mamo A, Cavallone L, Tuzmen S, Chabot C, Ferrario C, Hassan S, Edgren H, Kallioniemi O, Aleynikova O, Przybytkowski E, Malcolm K, Mousses S, Tonin PN, Basik M: An integrated genomic approach identifies ARID1A as a candidate tumor-suppressor gene in breast cancer. Oncogene 2012;31:2090-2100.

50 Kim M, Lu F, Zhang Y: Loss of HDAC-Mediated Repression and Gain of NF-kappaB Activation Underlie Cytokine Induction in ARID1A- and PIK3CA-Mutation-Driven Ovarian Cancer. Cell Rep 2016;17:275-288.

51 Walter DM, Venancio OS, Buza EL, Tobias JW, Deshpande C, Gudiel AA, Kim-Kiselak C, Cicchini M, Yates TJ, Feldser DM: Systematic In vivo Inactivation of Chromatin-Regulating Enzymes Identifies Setd2 as a Potent Tumor Suppressor in Lung Adenocarcinoma. Cancer Res 2017;77:1719-1729.

52 Shigetomi H, Oonogi A, Tsunemi T, Tanase Y, Yamada Y, Kajihara H, Yoshizawa Y, Furukawa N, Haruta S, Yoshida S, Sado T, Oi H, Kobayashi H: The role of components of the chromatin modification machinery in carcinogenesis of clear cell carcinoma of the ovary (Review). Oncol Lett 2011;2:591-597. 